\title{
Learning motivational significance of visual cues for reward schedules requires rhinal cortex
}

\author{
Zheng Liu, Elisabeth A. Murray and Barry J. Richmond \\ Laboratory of Neuropsychology, National Institute of Mental Health, National Institute of Health, Bethesda, Maryland 20892, USA \\ Correspondence should be addressed to B.J.R. (bjr@ln.nimh.nih.gov)
}

The limbic system is necessary to associate stimuli with their motivational and emotional significance. The perirhinal cortex is directly connected to this system, and neurons in this region carry signals related to a monkey's progress through visually cued reward schedules. This task manipulates motivation by displaying different visual cues to indicate the amount of work remaining until reward delivery. We asked whether rhinal (that is, entorhinal and perirhinal) cortex is necessary to associate the visual cues with reward schedules. When faced with new visual cues in reward schedules, intact monkeys adjusted their motivation in the schedules, whereas monkeys with rhinal cortex removals failed to do so. Thus, the rhinal cortex is critical for forming associations between visual stimuli and their motivational significance.

Motivation is defined as the incentive to act. It is generally believed that a limbic-striatal-pallidal circuit underlies the translation of motivation to action ${ }^{1-3}$. For example, the basolateral amygdala, a part of the limbic system, is important for conveying to the ventral striatum associative information concerning the environmental stimuli that predict rewards, which constitute goals of behavior ${ }^{4}$. In addition, electrophysiological studies show that neurons in the ventral striatum are active in relation to the motivational significance of visual cues ${ }^{5,6}$. In practice, motivational levels must be adjusted in response to sensory stimuli and events, to yield appropriate and adaptive goal-directed behavior. That is, information about the identity of a stimulus must be translated into a signal about its motivational significance. This led us ${ }^{7}$ to investigate where in the visual system such a transformation might occur. They recorded from neurons in the inferior temporal visual cortex of awake, behaving monkeys and found that in the perirhinal subdivision of the inferior temporal cortex, as in the ventral striatum, neurons carry signals about the motivational significance of visual cues. Based on these findings, the most ventromedial portion of the inferior temporal cortex, namely the rhinal (that is, the perirhinal and entorhinal) cortex, might be considered an extension of the limbic-striatal-pallidal circuitry involved in translating motivation to action.

Although these findings ${ }^{7}$ indicate that the rhinal cortex contains information about the motivational significance of visual cues, they cannot tell us whether the region is essential for assigning this significance. Accordingly, the present study tested whether the rhinal cortex is necessary for monkeys to learn about the motivational significance of visual cues. We used a version of the same behavioral task used in previous physiological studies ${ }^{5-7}$, visually cued reward schedules, that manipulates motivation by varying the amount of work required to gain a juice reward. In this task, different visual cues indicate the amount of work remaining until reward delivery, and, based solely on those visual cues, monkeys adjust their error rates to reflect the progress toward that reward. This behavioral phenomenon, the strong relationship of error rates to the 'distance' from reward, gave the opportunity to examine the monkeys' ability to acquire information about the motivational significance of visual cues.

In this study, monkeys performed schedules of 1, 2 or 3 trials, which were randomly interleaved within test sessions. On each trial, a monkey was required to release a lever when it detected a color change (from red to green) in the visual target stimulus presented on a screen; for simplicity, we refer to these trials as 'color discrimination' trials. Although the monkey was not punished for an incorrect trial, it had to perform a trial correctly to advance through the schedule. Only when the monkey had successfully completed the last trial in the schedule did it receive a liquid reward. In addition to the colored visual target stimulus, another stimulus, a grayscale rectangle serving as a cue to progress through the schedule, also appeared on the screen throughout each trial. The brightness of this cue indicated the number of trials remaining until reward would be delivered. For example, a bright cue signaled to the monkey that it was doing the first trial in a three-trial schedule. When that trial was completed, the stimuli disappeared, an intertrial interval ensued, and then a darker cue appeared, signaling to the monkey that it could now perform the second trial in the three-trial schedule. Finally, the darkest cue signaled the third and last trial. Although the cue provided no information relevant to performance of the color discrimination task, the monkeys adjusted their behavior in relation to the cue's brightness. Indeed, after only a few days of experience, the monkey's error rates reflected the number of trials remaining in the schedule until reward delivery, information that could only be gleaned from the cue's brightness. Specifically, the lowest error rates occurred on rewarded trials and progressively higher error rates were evident on trials further from delivery of reward. Thus, in this task, motivational levels were systematical- 
Fig 1. Visually cued reward schedule task. (a) Behavioral testing situation. The monkey sat in a primate chair facing a rear projection screen $\left(90^{\circ} \times 90^{\circ}\right)$ located $57 \mathrm{~cm}$ away. A touch lever was available to register responses. A black and white random dot background covered the whole screen. A cue (gray rectangle) and a colored dot were at the center of the screen. (b) D etailed timing of the sequence of events in a visually cued two-trial reward schedule. In each trial, the monkey was required to release a touch lever when a dot $\left(0.5^{\circ}\right)$ on the screen changed color from red to green. The monkey was required to perform correctly two of these color discrimination trials to obtain a reward at the end of the second trial. Each trial was assigned a 'schedule state' (current trial number/schedule length). The monkey started each trial by contacting a touch lever. Immediately after the lever was contacted, a visual cue was displayed and remained on without changing throughout the whole trial. The gray rectangle was the cue for schedule state $1 / 2$, whereas the dark rectangle was the cue for $2 / 2$. The cue was displayed for $900-1000$ $\mathrm{ms}$ before the trial progressed to the color discrimination phase. In the color discrimination phase of the trial, a red dot appeared at the center of the screen. After a rando mly selected wait time $(400,600$ $800,1000$ or $1200 \mathrm{~ms})$, the color of the dot changed from red to green, indicating that the monkey could release the lever to complete a trial. If the monkey released the lever within $1000 \mathrm{~ms}$, the dot changed from green to blue, signaling the monkey that a correct trial had been performed. After the blue dot was displayed for $150 \mathrm{~ms}$, all stimuli disappeared. If the trial was the last trial in a schedule, a liquid reward was delivered. If the monkey released the lever in the red dot period or in less than $200 \mathrm{~ms}$ after the onset of the green dot, or if the monkey did not release the lever within $1000 \mathrm{~ms}$ after the onset of the green dot, all stimuli disappeared, the trial was terminated, and an error was registered. Each trial was separated by a 1200-ms intertrial interval. (c) Brightness cues. (d) Length cues. In parentheses, schedule states for both brightness cues and length cues.

ly manipulated by using visual cues. The cues' influence on behavior was independent of the precise physical attributes or identity of the cues. Rather, the cues' influence arose because the monkey associated each cue with the number of trials remaining before a reward was obtained.

As indicated above, recordings were taken from single neurons in the inferior temporal cortex of monkeys performing a variant of this task ${ }^{7}$. The monkeys' error rates showed the same pattern as described earlier. All neurons in perirhinal cortex that showed responses to the rectangle cue showed responses that were selective for some part of the trial schedule. For example, some of the perirhinal neurons responded in the first trial of any schedule, whereas other neurons responded only in the last trial of any schedule, and still others responded selectively in one trial of a specific schedule. By contrast, neurons in area TE, the laterally adjacent region of the inferior temporal cortex, exhibited responses related only to stimulus identity. Based on these observations, we suggested that neurons in perirhinal cortex, but not in area TE, carry signals related to the motivational significance of visual cues.

Anatomically, the perirhinal cortex is at the interface of the ventral visual stream (the 'what' pathway) and the limbic system. It is strongly connected to area $\mathrm{TE}^{8,9}$, a higher-order neocortical field devoted to visual information processing ${ }^{10}$, as well as to parts of the limbic-striatal-pallidal regions (for example, ventral striatum $^{11-14}$ and amygdala ${ }^{15-17}$ ) thought to be important for translating motivation to action ${ }^{1,3}$. Thus, our physiological result ${ }^{7}$, combined with the anatomical evidence, led us to propose that the rhinal cortex is critical for acquiring and/or maintaining the relationship between a visual stimulus and its motivational significance. Using visually cued reward schedules, we show that after rhinal cortex removals, monkeys are impaired in using a preoper- a

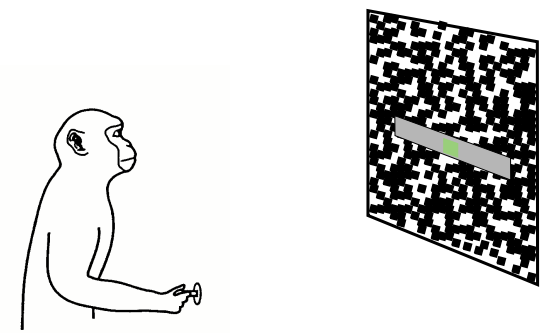

b

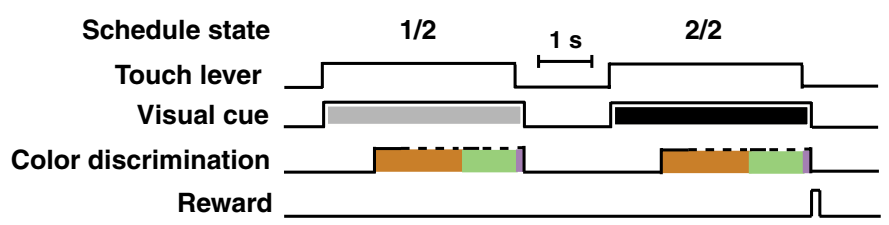

C

$(1 / 2)$

$(2 / 3)$

$(1 / 1,2 / 2,3 / 3)$

d

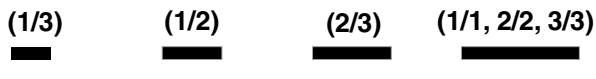

atively learned set of visual cues, and cannot acquire the association between new visual cues and their motivational significance.

\section{RESULTS}

Five adult rhesus monkeys (Macaca mulatta), from 4.5 to $5.5 \mathrm{~kg}$, were trained to perform 3 randomly mixed, visually cued reward schedules $^{5,6}$ (1-trial, 2-trial and 3-trial schedules; Fig. 1). Each trial in a given schedule could be referred to by a fraction describing the 'schedule state' (the trial position in a schedule/the schedule length). For example, the first, second and third trials in a 3-trial schedule had schedule states of $1 / 3,2 / 3$ and $3 / 3$, respectively. The progress through a schedule was indicated by a cue (a grayscale rectangle) presented on a screen. The brightness of the cue varied from white to black in direct proportion to the fractional value of the schedule state, so the schedule states $1 / 3,1 / 2$, and $2 / 3$ had distinct cues, whereas $1 / 1,2 / 2$, and $3 / 3$ had the same cue.

When the monkeys did $90 \%$ or more of the color discrimination trials correctly, the visually cued reward schedules were abruptly introduced. Although the monkeys were free to ignore the cues, the error rates for the different schedule states started to diverge in the first test session after the schedules were introduced. This trend continued, so that after the initial two or three sessions, the error rates were strongly related to the schedule states (Fig. 2a). In this sense, their behavior stabilized at this stage of the experiment ${ }^{5-7}$. The end of a schedule was signaled only by the cue, and as the end approached, the monkeys made fewer errors. For each of five monkeys, the error rates were the same in all rewarded schedule states (that is, $1 / 1,2 / 2$ and $3 / 3$ ), no matter which schedule (1-, 2- or 3-trial) was ending $\left(\chi^{2}(2)=5.4,2.0\right.$, $0.85,0.77$ and $2.5 ; p=0.06,0.36,0.67,0.68$ and 0.28 for monkeys Rh1, Rh2, Rh3, Con1 and Con2, respectively). Therefore, the data from all three rewarded trial types were combined.

After the monkeys' error rates had stabilized in this standard 
Fig. 2. Error rates of the monkeys ( $n=5$ ) performing the visually cued reward schedules using brightness cues. (a) Cued condition showing error rates for each schedule state. (b) Cued and shuffled conditions with error rates for rewarded trials combined. Error bars, s.e.m. In the cued condition, the monkeys made fewer errors as the cues indicated that the trials were closer to reward delivery. The monkeys made the fewest errors, overall, in the shuffled condition.

'cued' condition, a 'shuffled' condition was introduced. Whereas in the cued condition, the visual cues reliably indicated the progress through the schedules, in the shuffled condition, the cues were randomly assigned so they no longer related to progress through the schedules. The cued and shuffled trials were given in half-session blocks; the conditions were switched without notice. The order of testing on the cued and shuffled conditions was alternated on a daily basis to control for sequence effects. For each intact monkey, the error rates in the cued condition were significantly different across all schedule states considered as a group $\left(\chi^{2}(3)=128.9,47.9,210.8,114.6\right.$ and $32.1 ; p=0.00001$ for all five monkeys (Table 1 ), as well as across the three nonrewarded states $(1 / 3,1 / 2$ and $2 / 3$ states $)$ considered as a group $\left(\chi^{2}(2)=30.6,27.6,13.5,7.9\right.$ and $6.7 ; p=0.00001,0.00001,0.001$, 0.01 and 0.04 for monkeys Rh1, Rh2, Rh3, Con1 and Con2, respectively). In addition, the mean error rates covaried with the schedule states; that is, there was a strong linear relationship between the mean error rate and the brightness of the cues (linear regression, $r^{2}=0.98, F_{1,3}=133.4, p=0.007$; Fig. 2 b). In contrast, in the shuffled condition, the monkeys' error rates did not vary with brightness of the cues (one-way ANOVA, $F_{3,19}=0.2$, $p=0.89$; Fig. 2b). The monkeys made the fewest errors in the shuffled condition (error rate, $4.9 \pm 0.3 \%$, mean \pm s.e.m., $n=20$ ). These results are similar to those reported previously ${ }^{5,6}$. One difference from our earlier findings is that there was no relationship between mean reaction times and schedule states in this group of monkeys. This difference might be related to the monkeys' ages; the monkeys in the present study were smaller (4 to $5 \mathrm{~kg}$ ) than those used in the previous physiology studies $(>6 \mathrm{~kg})$, so it is likely that they were also younger.

The pattern of error rates across schedule states, in which each cue is assigned its own significance, was the most important measure in this task. That is, the linear relationship between the error rates and schedule states allowed us to infer that the

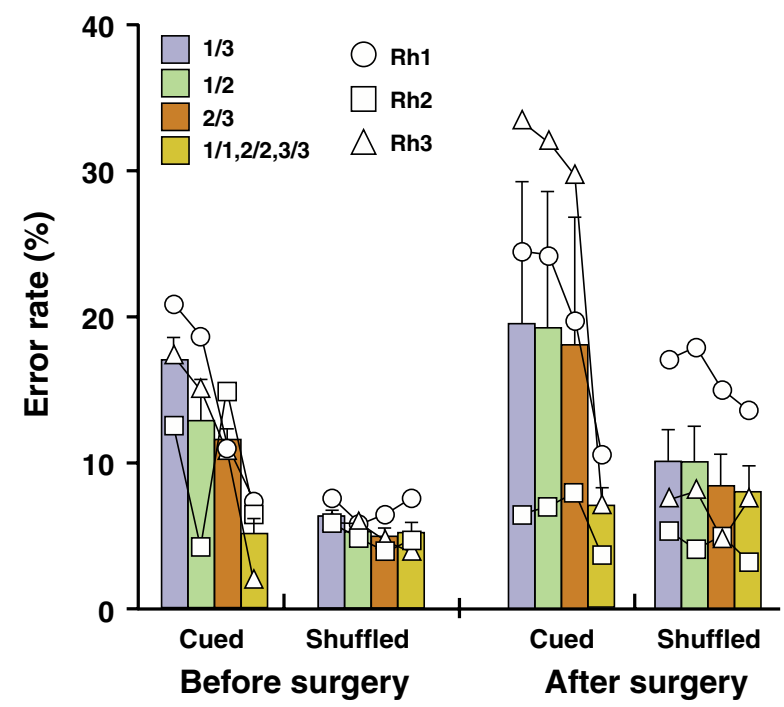

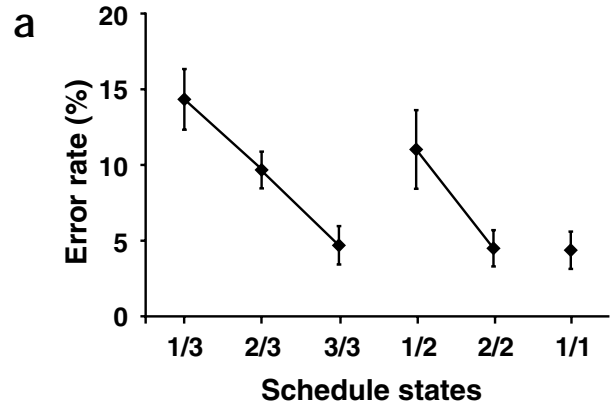

b

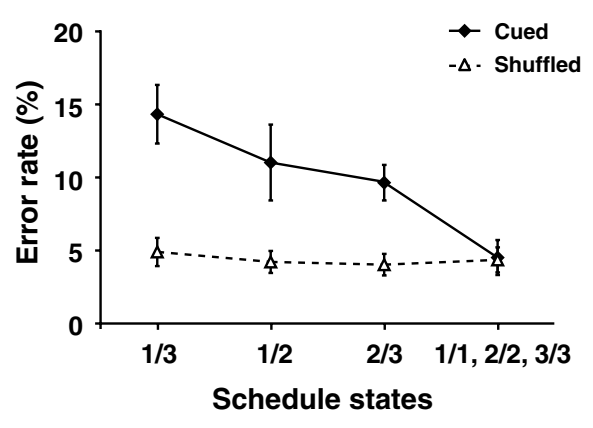

monkeys' motivational levels were being systematically manipulated by the brightness cues. A different pattern of results (for example, one in which the error rate for a single schedule state differed from the remaining ones) might have been due to a simple bias in responding, or superstitious behavior unrelated to motivational levels.

To determine whether the rhinal cortex is needed to maintain the normal association between the visual cues and monkeys' motivational levels, three of the five monkeys were given one-stage, bilateral aspiration removals of rhinal cortex, and were then assessed for their retention of the pre-operatively used brightness cues. For the three monkeys with rhinal cortex removals, the brightness cues had roughly the same relationship to the mean error rates as they had before surgery (interaction term of a two-way ANOVA, $F_{3,23}=0.17, p=0.92$; Fig. 3; Table 1). However, the similarity suggested by the ANOVA was due entirely to the relationship between rewarded and nonrewarded trials. If the nonrewarded trials were considered alone, there was a significant effect of the rhinal cortex removal. Specifically, whereas the error rates for the different types of nonrewarded trials $(1 / 3,1 / 2$ and $2 / 3$ schedule states) were significantly different before surgery $\left(\chi^{2}(2)=30.6,27.7\right.$ and $13.5 ; p=0.0001,0.0001$ and 0.001 for Rh1, Rh2 and Rh3, respectively), they were statistically indistinguishable after the surgery $\left(\chi^{2}(2)=5.6,0.87\right.$ and $2.3 ; p=0.06,0.87$ and 0.32$)$. In addition, whereas there was a strong linear relationship between the mean error rates and the brightness of the cues before surgery (linear regression, $r^{2}=0.97$,

Fig. 3. Error rates of monkeys performing the visually cued reward schedules using the brightness cues before and after bilateral removal of the rhinal cortex. Error bars, s.e.m. The visual cues affected the monkeys' behavior both before and after the surgery. However, after the surgery, the linear relationship between the error rates and the schedule states disappeared, and the overall error rate was increased by about $4.9 \%$. 
Table 1. Percent error rates of the monkeys performing visually cued reward schedules using the brightness cues before and after rhinal cortex removal.

\begin{tabular}{|c|c|c|c|c|c|c|}
\hline Monkey & Schedule state & Trial type & Befor & urgery & Afte & Irgery \\
\hline Rh1 & $\begin{array}{l}1 / 3 \\
1 / 2 \\
2 / 3 \\
1 / 1,2 / 2,3 / 3\end{array}$ & $\begin{array}{l}C \\
E \\
R(\%) \\
C \\
E \\
R(\%) \\
C \\
E \\
R(\%) \\
C \\
E \\
R(\%)\end{array}$ & $\begin{array}{c}\text { Cued } \\
630 \\
164 \\
\mathbf{2 0 . 7} \\
667 \\
155 \\
\mathbf{1 8 . 9} \\
630 \\
74 \\
\mathbf{1 0 . 5} \\
1958 \\
160 \\
\mathbf{7 . 6}\end{array}$ & $\begin{array}{c}\text { Shuffled } \\
731 \\
59 \\
\mathbf{7 . 5} \\
732 \\
44 \\
\mathbf{5 . 7} \\
733 \\
51 \\
\mathbf{6 . 5} \\
678 \\
55 \\
\mathbf{7 . 5}\end{array}$ & $\begin{array}{c}\text { Cued } \\
509 \\
164 \\
\mathbf{2 4 . 4} \\
555 \\
176 \\
\mathbf{2 4 . 1} \\
509 \\
123 \\
\mathbf{1 9 . 5} \\
1575 \\
190 \\
\mathbf{1 0 . 8}\end{array}$ & $\begin{array}{c}\text { Shuffled } \\
593 \\
123 \\
\mathbf{1 7 . 2} \\
584 \\
127 \\
\mathbf{1 7 . 9} \\
621 \\
110 \\
\mathbf{1 5 . 0} \\
616 \\
97 \\
\mathbf{1 3 . 6}\end{array}$ \\
\hline Rh2 & $\begin{array}{l}1 / 3 \\
1 / 2 \\
2 / 3 \\
1 / 1,2 / 2,3 / 3\end{array}$ & $\begin{array}{l}C \\
E \\
\mathbf{R}(\%) \\
C \\
E \\
\mathbf{R}(\%) \\
C \\
E \\
\mathbf{R}(\%) \\
C \\
E \\
\mathbf{R}(\%)\end{array}$ & $\begin{array}{r}376 \\
53 \\
\mathbf{1 2 . 4} \\
398 \\
17 \\
\mathbf{4 . 1} \\
374 \\
64 \\
\mathbf{1 4 . 6} \\
1200 \\
81 \\
\mathbf{6 . 3}\end{array}$ & $\begin{array}{r}640 \\
39 \\
\mathbf{5 . 7} \\
650 \\
32 \\
\mathbf{4 . 7} \\
658 \\
26 \\
3.8 \\
667 \\
33 \\
\mathbf{4 . 7}\end{array}$ & $\begin{array}{r}486 \\
34 \\
\mathbf{6 . 5} \\
557 \\
41 \\
\mathbf{6 . 9} \\
487 \\
42 \\
\mathbf{7 . 9} \\
1568 \\
62 \\
\mathbf{3 . 8}\end{array}$ & $\begin{array}{r}637 \\
37 \\
\mathbf{5 . 5} \\
674 \\
29 \\
\mathbf{4 . 1} \\
682 \\
35 \\
\mathbf{4 . 9} \\
654 \\
18 \\
\mathbf{2 . 7}\end{array}$ \\
\hline Rh3 & $\begin{array}{l}1 / 3 \\
1 / 2 \\
2 / 3 \\
1 / 1,2 / 2,3 / 3\end{array}$ & $\begin{array}{l}C \\
E \\
R(\%) \\
C \\
E \\
R(\%) \\
C \\
E \\
R(\%) \\
C \\
E \\
R(\%)\end{array}$ & $\begin{array}{r}538 \\
113 \\
\mathbf{1 7 . 4} \\
581 \\
101 \\
\mathbf{1 4 . 8} \\
539 \\
61 \\
\mathbf{1 0 . 2} \\
1702 \\
29 \\
\mathbf{1 . 7}\end{array}$ & $\begin{array}{r}653 \\
40 \\
\mathbf{5 . 8} \\
597 \\
37 \\
\mathbf{5 . 8} \\
621 \\
29 \\
\mathbf{4 . 5} \\
704 \\
25 \\
\mathbf{3 . 4}\end{array}$ & $\begin{array}{r}371 \\
186 \\
\mathbf{3 3 . 4} \\
407 \\
189 \\
\mathbf{3 1 . 7} \\
370 \\
152 \\
\mathbf{2 9 . 1} \\
1221 \\
87 \\
\mathbf{6 . 7}\end{array}$ & $\begin{array}{r}547 \\
45 \\
\mathbf{7 . 6} \\
513 \\
45 \\
\mathbf{8 . 1} \\
539 \\
27 \\
\mathbf{4 . 8} \\
542 \\
43 \\
\mathbf{7 . 4}\end{array}$ \\
\hline Conl & $\begin{array}{l}1 / 3 \\
1 / 2 \\
2 / 3 \\
1 / 1,2 / 2,3 / 3\end{array}$ & $\begin{array}{l}C \\
E \\
R(\%) \\
C \\
E \\
R(\%) \\
C \\
E \\
R(\%) \\
C \\
E \\
R(\%)\end{array}$ & $\begin{array}{r}656 \\
75 \\
\mathbf{1 0 . 3} \\
732 \\
71 \\
\mathbf{8 . 8} \\
654 \\
43 \\
\mathbf{6 . 2} \\
2139 \\
38 \\
\mathbf{1 . 7}\end{array}$ & $\begin{array}{r}1029 \\
20 \\
\mathbf{1 . 9} \\
957 \\
22 \\
\mathbf{2 . 2} \\
924 \\
19 \\
\mathbf{2 . 0} \\
1004 \\
26 \\
\mathbf{2 . 5}\end{array}$ & & \\
\hline Con2 & $\begin{array}{l}1 / 3 \\
1 / 2 \\
2 / 3 \\
1 / 1,2 / 2,3 / 3\end{array}$ & $\begin{array}{l}C \\
E \\
R(\%) \\
C \\
E \\
R(\%) \\
C \\
E \\
R(\%) \\
C \\
E \\
R(\%)\end{array}$ & $\begin{array}{r}694 \\
86 \\
\mathbf{1 1 . 0} \\
710 \\
66 \\
\mathbf{8 . 5} \\
694 \\
55 \\
\mathbf{7 . 3} \\
2075 \\
116 \\
\mathbf{5 . 3}\end{array}$ & $\begin{array}{r}906 \\
34 \\
\mathbf{3 . 6} \\
921 \\
25 \\
\mathbf{2 . 6} \\
892 \\
31 \\
\mathbf{3 . 4} \\
906 \\
34 \\
\mathbf{3 . 6}\end{array}$ & & \\
\hline
\end{tabular}


Fig. 4. Error rates of the monkeys performing the visually cued reward schedules using the brightness or length cues, after the cues were introduced. (a) Mean error rates of five monkeys performing the schedules using the brightness cues. (b) Mean error rates of two control monkeys performing the schedules using the length cues. The error rates were significantly different across schedule states, and there was a linear relationship between error rates and schedule states, evident from the second week onward. (c) Mean error rates of the three monkeys after rhinal cortex removals, performing the schedules using the length cues. There is no linear relationship between the error rates and the schedule states at any point in time. Compare with (b).

$F_{1,3}=61.6, p=0.01$ ), this linear relationship was no longer significant after surgery (linear regression, $r^{2}=0.88, F_{1,3}=14.8$, $p=0.06$ ). Just as before surgery, the error rates in the shuffled condition were not related to the cue (one-way ANOVA, $F_{3,11}=0.1, p$ $=0.95$ ). Removal of the rhinal cortex significantly increased the error rate by an average of $4.5 \%$ across all conditions (before surgery, $8.5 \pm 1.1 \%$, mean \pm s.e.m.; after surgery, $13.0 \pm 1.9 \%$; $n=24$; paired $t$-test, $t_{23}=1.7 ; p=0.001$ ).

To determine the effect of rhinal cortex removal on learning about the motivational significance of new cues, we compared the acquisition of the cues learned before surgery with acquisition of a set of cues introduced after surgery (Fig. 4a; Table 2). As already indicated, the five intact monkeys rapidly learned the original set of cues. Four of the monkeys developed a significant linear relationship between the error rates and schedule states in the very first week after the cues were introduced (linear regression, $r^{2}=0.91$, 0.90, 0.90 and $0.97, F_{1,3}=19.8,19.0,19.1$ and $67.8, p=0.04,0.04$, 0.04 and 0.01 , for monkeys Rh1, Rh3, Con 1 and Con2, respectively). Monkey Rh2 developed the same relationship in the second week (linear regression, $r^{2}=0.97, F_{1,3}=62.4, p=0.01$ ). The relationship between the error rates and schedule states remained the same for the second to the fourth week (interaction term of a two-way ANOVA, $F_{6,48}=0.07, p=0.99$ ).

For the three operated monkeys, the length cues were introduced immediately after the postoperative testing with the brightness cues. All of the monkeys were tested for six weeks. In marked contrast to the intact monkeys, the error rates averaged across the three operated monkeys showed no significant difference at any point for any schedule state in the six weeks of exposure (Table 2; Fig. 4c; one-way ANOVA, $F_{3,11}=0.16,0.07,0.05,3.4$ and $0.16, p=0.92,0.97,0.98,0.54,0.07$ and 0.92 for week 1 to 6 , respectively). Thus, the behavior of the operated monkeys was not affected by the new length cues. During the sixth week of testing, the group error rates were still indistinguishable from the error rates seen in the same monkeys performing the schedules

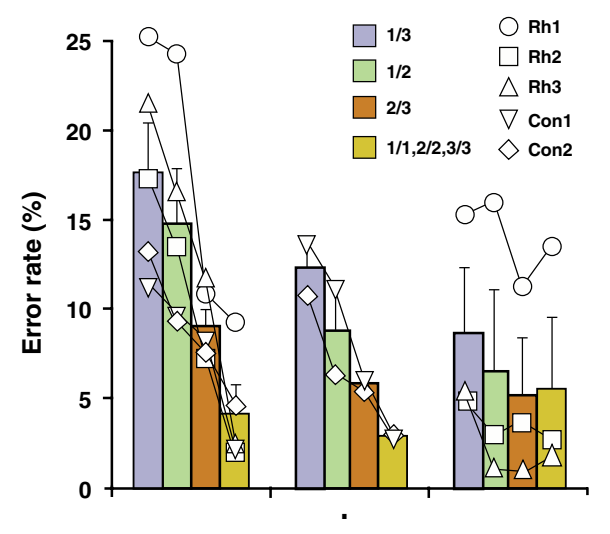

a

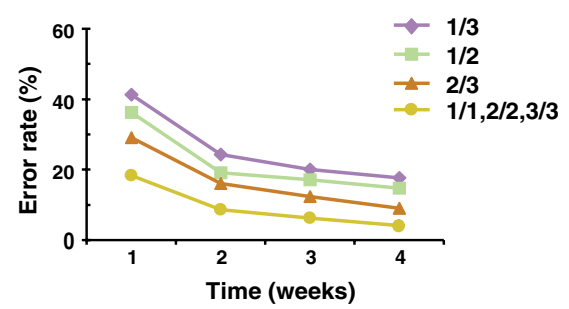

b

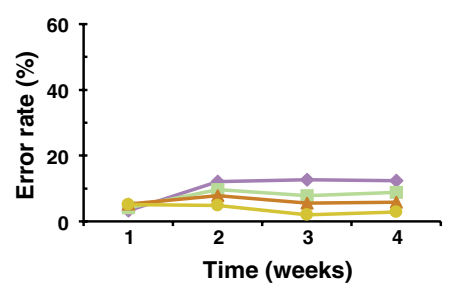

C

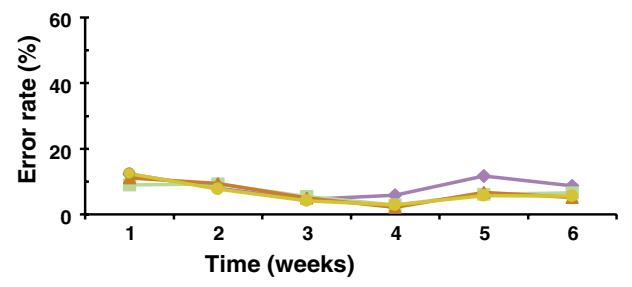

using the shuffled brightness cues preoperatively (Fig. 5 ; interaction term of a two-way ANOVA, $F_{3,23}=0.06, p=0.98$ ). Two monkeys, Rh1 and Rh2, were tested again for 3 weeks after having been 'on rest' for 2 and 30 weeks, respectively. Of these monkeys, Rh1 (at the thirty-first week) and Rh3 (at the fourth week), made more errors in the $1 / 3$ condition than in the other states (Table $2 ; \chi^{2}(3)=58.1$ and 27.0, $p=0.00001$ and 0.00001 respectively). No monkeys, however, developed the significant linear relationship between the error rates and schedule states during 6 weeks of testing (linear regression, $r^{2}=0.08,0.06,0.3,0.41$, 0.55 and $0.62, F_{1,3}=0.16,0.12,0.88,1.41,2.49$ and 3.34 , $p=0.8,0.83,0.64,0.59,0.53$ and 0.51 , for weeks 1 to 6 , respectively), as had the controls (Fig. 4), nor did the two monkeys given additional testing develop the relationship (Table 2; linear regression, $r^{2}=0.42,0.56$ and $0.48, F_{1,3}=1.45,2.57$, and 1.85, $p=0.35,0.25$ and 0.31 , for weeks $\mathrm{A}, \mathrm{B}$ and $\mathrm{C}$, respectively).

Two additional analyses were undertaken to help interpret the results from the main task. First, in performing visually cued reward schedules, monkeys made statistically indistinguishable numbers of errors by releasing the touch-lever too early or too late $\left(\chi^{2}\right.$ test, $\left.p>0.05\right)$ in all testing conditions, both before and after surgery. Second, all monkeys were tested to determine whether they could detect the change in either the brightness or length of the cue rectangle in place of the color discrimination
Fig. 5. Error rates of the monkeys performing the visually cued reward schedules using the brightness or length cues in the final testing week. (a) Error rates for the all monkeys ( $n=5$ ) performing the schedules using the brightness cues during the fourth week of testing, before the rhinal cortex removals. (b) Error rates for the control monkeys $(n=2)$ performing the schedules using the length cues during the fourth week of testing. (c) Error rates for the monkeys with rhinal cortex removals $(n=3)$ performing the schedules using the length cues during the sixth week of testing. There is a significant linear relationship between the error rates and the schedule states for the data in (a) and (b), but not (c). 


\begin{tabular}{|c|c|c|c|c|c|c|c|c|c|c|c|c|c|c|c|c|}
\hline \multirow[t]{3}{*}{ Monkey } & \multirow{3}{*}{$\begin{array}{l}\text { Schedule } \\
\text { state }\end{array}$} & \multirow{3}{*}{$\begin{array}{l}\text { Trial } \\
\text { type }\end{array}$} & \multicolumn{4}{|c|}{ Brightness cue } & \multicolumn{10}{|c|}{ Length cue } \\
\hline & & & \multicolumn{4}{|c|}{ Trials in week } & \multicolumn{10}{|c|}{ Trials in week } \\
\hline & & & 1 & 2 & 3 & 4 & 1 & 2 & 3 & 4 & 5 & 6 & 7 & 8 & 9 & 10 \\
\hline \multirow{12}{*}{ Rh1 } & $1 / 3$ & C & 584 & 618 & 336 & 337 & 369 & 430 & 563 & 607 & 498 & 474 & 341 & 353 & 603 & 530 \\
\hline & & $\mathrm{E}$ & 491 & 185 & 52 & 114 & 100 & 62 & 49 & 21 & 64 & 89 & 40 & 58 & 81 & 113 \\
\hline & & R (\%) & 45.7 & 23.0 & 13.4 & 25.3 & 21.3 & 12.6 & 8.0 & 3.3 & 11.4 & 15.8 & 10.5 & 14.1 & 11.8 & 17.6 \\
\hline & $1 / 2$ & C & 602 & 621 & 351 & 345 & 368 & 455 & 551 & 624 & 560 & 498 & 385 & 358 & 705 & 596 \\
\hline & & $\mathrm{E}$ & 363 & 136 & 34 & 113 & 69 & 73 & 64 & 25 & 50 & 95 & 15 & 36 & 35 & 45 \\
\hline & & R (\%) & 37.6 & 18.0 & 8.8 & 24.7 & 15.8 & 13.8 & 10.4 & 3.9 & 8.2 & 16.0 & 3.8 & 9.1 & 4.7 & 7.0 \\
\hline & $2 / 3$ & C & 584 & 618 & 335 & 337 & 371 & 429 & 563 & 607 & 501 & 475 & 341 & 355 & 605 & 531 \\
\hline & & $\mathrm{E}$ & 172 & 89 & 39 & 41 & 71 & 67 & 61 & 19 & 60 & 64 & 14 & 32 & 16 & 28 \\
\hline & & R (\%) & 22.8 & 12.6 & 10.4 & 10.8 & 16.1 & 13.5 & 9.8 & 3.0 & 10.7 & 11.9 & 3.9 & 8.3 & 2.6 & 5.0 \\
\hline & $1 / 1,2 / 2,3 / 3$ & $C$ & 1745 & 1863 & 1043 & 1039 & 1114 & 1299 & 1701 & 1845 & 1596 & 1487 & 1072 & 1075 & 1945 & 1688 \\
\hline & & $E$ & 353 & 189 & 85 & 112 & 231 & 158 & 156 & 85 & 143 & 225 & 46 & 105 & 81 & 105 \\
\hline & & R (\%) & 16.8 & 9.2 & 7.5 & 9.7 & 17.2 & 10.8 & 8.4 & 4.4 & 8.2 & 13.1 & 4.1 & 8.9 & 4.0 & 5.9 \\
\hline \multirow[t]{12}{*}{ Rh2 } & $1 / 3$ & C & 319 & 334 & 370 & 408 & 294 & 484 & 383 & 523 & 658 & 494 & 334 & 373 & 538 & 458 \\
\hline & & $\mathrm{E}$ & 56 & 122 & 111 & 86 & 11 & 12 & 2 & 7 & 89 & 27 & 11 & 9 & 13 & 12 \\
\hline & & R (\%) & 14.9 & 26.8 & 23.1 & 17.4 & 3.6 & 2.4 & 0.5 & 1.3 & 11.9 & 5.2 & 3.2 & 2.4 & 2.4 & 2.6 \\
\hline & $1 / 2$ & $C$ & 322 & 323 & 387 & 460 & 361 & 597 & 516 & 658 & 658 & 546 & 399 & 416 & 612 & 522 \\
\hline & & $E$ & 68 & 111 & 117 & 70 & 8 & 13 & 7 & 9 & 46 & 16 & 5 & 9 & 12 & 4 \\
\hline & & R (\%) & 17.4 & 25.6 & 23.2 & 13.2 & 2.2 & 2.1 & 1.3 & 1.3 & 6.5 & 2.8 & 1.2 & 2.1 & 1.9 & 0.8 \\
\hline & $2 / 3$ & C & 317 & 332 & 370 & 406 & 294 & 483 & 383 & 522 & 660 & 495 & 334 & 372 & 537 & 456 \\
\hline & & $E$ & 100 & 92 & 53 & 31 & 19 & 14 & 7 & 7 & 47 & 17 & 13 & 10 & 21 & 37 \\
\hline & & R (\%) & 24.0 & 21.7 & 12.5 & 7.1 & 6.1 & 2.8 & 1.8 & 1.3 & 6.6 & 3.3 & 3.7 & 2.6 & 3.8 & 7.5 \\
\hline & $1 / 1,2 / 2,3 / 3$ & C & 952 & 963 & 1105 & 1294 & 1002 & 1643 & 1385 & 1830 & 1949 & 1610 & 1105 & 1197 & 1735 & 1484 \\
\hline & & $E$ & 301 & 215 & 98 & 27 & 52 & 63 & 11 & 33 & 119 & 42 & 17 & 21 & 45 & 23 \\
\hline & & R (\%) & 24.0 & 18.3 & 8.1 & 2.0 & 4.9 & 3.7 & 0.8 & 1.8 & 5.8 & 2.5 & 1.5 & 1.7 & 2.5 & 1.5 \\
\hline \multirow[t]{12}{*}{$\mathrm{Rh} 3$} & $1 / 3$ & C & 319 & 369 & 271 & 270 & 410 & 472 & 410 & 234 & 197 & 263 & & & & \\
\hline & & $E$ & 254 & 81 & 148 & 73 & 56 & 46 & 23 & 35 & 26 & 14 & & & & \\
\hline & & R (\%) & 44.3 & 18.0 & 35.3 & 21.3 & 12.0 & 8.9 & 5.3 & 13.0 & 11.7 & 5.1 & & & & \\
\hline & $1 / 2$ & $C$ & 362 & 408 & 287 & 301 & 497 & 485 & 384 & 258 & 239 & 327 & & & & \\
\hline & & $E$ & 239 & 74 & 142 & 61 & 49 & 65 & 18 & 9 & 9 & 2 & & & & \\
\hline & & R (\%) & 39.8 & 15.4 & 33.1 & 16.9 & 9.0 & 11.8 & 4.5 & 3.4 & 3.6 & 0.6 & & & & \\
\hline & $2 / 3$ & C & 320 & 368 & 271 & 272 & 411 & 474 & 406 & 234 & 197 & 263 & & & & \\
\hline & & $E$ & 108 & 58 & 68 & 36 & 51 & 64 & 14 & 5 & 6 & 1 & & & & \\
\hline & & R (\%) & 25.2 & 13.6 & 20.1 & 11.7 & 11.0 & 11.9 & 3.3 & 2.1 & 3.0 & 0.4 & & & & \\
\hline & $1 / 1,2 / 2,3 / 3$ & C & 1051 & 1178 & 837 & 890 & 1382 & 1430 & 1192 & 754 & 668 & 890 & & & & \\
\hline & & $E$ & 257 & 60 & 66 & 20 & 243 & 137 & 41 & 19 & 21 & 8 & & & & \\
\hline & & R (\%) & 19.6 & 4.8 & 7.3 & 2.2 & 15.0 & 8.7 & 3.3 & 2.5 & 3.0 & 0.9 & & & & \\
\hline \multirow[t]{12}{*}{ Con1 } & $1 / 3$ & C & 246 & 521 & 360 & 394 & 762 & 605 & 479 & 460 & & & & & & \\
\hline & & $E$ & 413 & 274 & 58 & 50 & 31 & 93 & 84 & 73 & & & & & & \\
\hline & & R (\%) & 62.7 & 34.5 & 13.9 & 11.3 & 3.9 & 13.3 & 14.9 & 13.7 & & & & & & \\
\hline & $1 / 2$ & C & 236 & 555 & 386 & 437 & 815 & 637 & 519 & 487 & & & & & & \\
\hline & & $\mathrm{E}$ & 286 & 185 & 47 & 47 & 44 & 79 & 49 & 62 & & & & & & \\
\hline & & R (\%) & 54.8 & 25.0 & 10.9 & 9.7 & 5.1 & 11.0 & 8.6 & 11.3 & & & & & & \\
\hline & $2 / 3$ & $C$ & 246 & 520 & 358 & 393 & 763 & 604 & 478 & 460 & & & & & & \\
\hline & & E & 283 & 154 & 37 & 35 & 53 & 53 & 32 & 30 & & & & & & \\
\hline & & R (\%) & 53.5 & 22.8 & 9.4 & 8.2 & 6.5 & 8.1 & 6.3 & 6.1 & & & & & & \\
\hline & $1 / 1,2 / 2,3 / 3$ & C & 715 & 1655 & 1160 & 1274 & 2324 & 1811 & 1504 & 1448 & & & & & & \\
\hline & & $\mathrm{E}$ & 190 & 98 & 21 & 28 & 163 & 110 & 38 & 43 & & & & & & \\
\hline & & R (\%) & 21.0 & 5.6 & 1.8 & 2.2 & 6.6 & 5.7 & 2.5 & 2.9 & & & & & & \\
\hline
\end{tabular}

in individual trials in a one-trial schedule. For all five monkeys, the brightness discrimination was examined at the end of all testing, and the length discrimination was tested in the middle of the testing of schedules with the length cues. All monkeys could readily detect and discriminate the brightness and the length differences between the two cue rectangles, even with the smallest difference (error rates, brightness, $12.7 \% \pm 0.5 \%$; length, $18.5 \% \pm 3.5 \%$; mean \pm s.e.m.; $n=5$ ).

\section{Discussion}

When the control monkeys were provided with a new set of cues to guide their behavior, a linear relationship between the 


\begin{tabular}{|c|c|c|c|c|c|c|c|c|c|c|c|c|c|c|c|c|}
\hline \multirow[t]{3}{*}{ Monkey } & \multirow{3}{*}{$\begin{array}{l}\text { Schedule } \\
\text { state }\end{array}$} & \multirow{3}{*}{$\begin{array}{l}\text { Trial } \\
\text { type }\end{array}$} & \multicolumn{4}{|c|}{ Brightness cue } & \multicolumn{10}{|c|}{ Length cue } \\
\hline & & & \multicolumn{4}{|c|}{ Trials in week } & \multicolumn{10}{|c|}{ Trials in week } \\
\hline & & & 1 & 2 & 3 & 4 & 1 & 2 & 3 & 4 & 5 & 6 & 7 & 8 & 9 & 10 \\
\hline \multirow[t]{12}{*}{ Con2 } & $1 / 3$ & C & 414 & 595 & 349 & 345 & 728 & 530 & 655 & 617 & & & & & & \\
\hline & & $E$ & 266 & 139 & 60 & 52 & 19 & 65 & 75 & 76 & & & & & & \\
\hline & & R (\%) & 39.1 & 18.9 & 14.7 & 13.1 & 2.5 & 10.9 & 10.3 & 11.0 & & & & & & \\
\hline & $1 / 2$ & C & 404 & 575 & 359 & 351 & 772 & 576 & 637 & 566 & & & & & & \\
\hline & & $E$ & 188 & 77 & 39 & 37 & 24 & 52 & 49 & 38 & & & & & & \\
\hline & & R (\%) & 31.8 & 11.8 & 9.8 & 9.5 & 3.0 & 8.3 & 7.1 & 6.3 & & & & & & \\
\hline & $2 / 3$ & C & 415 & 616 & 349 & 345 & 727 & 528 & 655 & 617 & & & & & & \\
\hline & & $E$ & 103 & 64 & 37 & 28 & 31 & 43 & 33 & 36 & & & & & & \\
\hline & & $\mathbf{R}(\%)$ & 19.9 & 9.4 & 9.6 & 7.5 & 4.1 & 7.5 & 4.8 & 5.5 & & & & & & \\
\hline & $1 / 1,2 / 2,3 / 3$ & C & 1200 & 1290 & 1038 & 1039 & 2252 & 1610 & 1968 & 1785 & & & & & & \\
\hline & & $E$ & 131 & 74 & 69 & 48 & 88 & 66 & 31 & 53 & & & & & & \\
\hline & & R (\%) & 9.8 & 5.4 & 6.2 & 4.4 & 3.8 & 3.9 & 1.6 & 2.9 & & & & & & \\
\hline
\end{tabular}

Rh1, Rh2 and Rh3 received rhinal cortex lesions. Con1 and Con2, unoperated control monkeys. W eeks 1 to 6 , six consecutive weeks of testing after the new cues were introduced to the monkeys. W eeks A, B and C, three additional weeks of testing after Rh1 was rested for 2 weeks and Rh2 was rested for 30 weeks after the initial 6 weeks of testing. $C$, number of trials done correctly; $E$, number of trials done incorrectly; $R$, error rate.

error rates and schedule states developed quickly. By contrast, the operated monkeys never developed this linear relationship in the time period we investigated. That is, unlike the intact controls, monkeys with rhinal cortex removals failed to adjust their motivational levels in response to a new set of visual cues that signaled progress through reward schedules. Furthermore, even though the monkeys with rhinal cortex removals did retain the association between a cue and immediate reward delivery, as evidenced by partial retention of the preoperatively-learned cues, they seemed unable to predict the amount of work needed to receive a deferred reward. These results indicate that the rhinal cortex is critical for forming and maintaining the associations between visual stimuli and their motivational significance.

A simple visual perceptual deficit is unlikely to be the cause of the impairment we observed, because the operated monkeys could distinguish among the cues. Consistent with this finding, monkeys with rhinal cortex removals are reported to learn visual discrimination problems at the same rate as unoperated controls ${ }^{18-22}$, especially when small stimulus sets are used. In addition, our monkeys with rhinal cortex removals made roughly equal numbers of early and late errors in their performance before and after surgery, making it unlikely that a change in general motor response times would explain the lack of influence of the length cues. Finally, it is possible that the deficit in learning the motivational significance of the length cues occurred because the operated monkeys were unable to switch away from the original rule, namely, that the cue's brightness was the feature signaling the amount of work until reward. However, even though monkeys with rhinal cortex damage make significantly more errors in object reversal learning than controls, they nonetheless learn the reversals with relatively few errors ${ }^{23}$. Thus, the possibility that a reversal impairment could explain our finding is unlikely.

In previous studies, damage to rhinal cortex has failed to impair visual discrimination learning for food reward ${ }^{18-22}$. In discrimination learning, the monkeys' correct responses always led to immediate delivery of reward, whereas here, the monkeys were required to carry out schedules of trials, and the reward was only given at the end of the schedule. Therefore, the direct association between a stimulus and reward, under conditions in which the motivational levels are assumed to be roughly constant, is largely unaffected by rhinal cortex lesions. By contrast, under conditions in which motivational levels are systematically manipulated, as in the present study, the rhinal cortex is essential for associating visual stimuli with progress through the reward schedules, a measure of the work remaining until reward is delivered. Motivational levels can also be manipulated by changing the value of rewards. In the context of a reinforcer devaluation task, rhinal cortex ablations fail to disrupt the association between a stimulus and the value of a reward ${ }^{21}$. Understanding the way in which different motivational factors guide behavior will be an important direction for future research.

The critical role of the rhinal cortex in attaching motivational significance to visual cues might seem surprising. However, the rhinal cortex is a crossroad between the ventral visual stream $^{8,9}$, a region important for processing visual stimulus identity $^{10}$, and the limbic-striatal-pallidal system ${ }^{11-17}$, a set of structures thought to be important for associating stimuli with their motivational and emotional significance ${ }^{1,3}$. In processing stimuli, the rhinal cortex is richly interconnected with area $\mathrm{TE}^{8,9}$ (which is modality specific for vision), other higher-order modality-specific cortical fields ${ }^{24}$, and multimodal processing areas $^{24}$. Damage to the rhinal cortex interferes with the acquisition and storage of information relating the different sensory qualities of individual objects ${ }^{18,25-37}$. Whether the rhinal cortex is necessary for learning the motivational significance of nonvisual cues has not yet been examined. The present results, together with the physiological findings ${ }^{7}$ that neurons in perirhinal cortex signal the motivational significance of visual cues, provide strong evidence that rhinal cortex is a critical part of the neural circuitry involved in translating motivation to action.

\section{Methods}

Behavioral task. The task consisted of three randomly interleaved visually cued reward schedules, which had one, two or three trials ${ }^{5,6}$. The reward schedules were built by requiring the monkeys to perform 1,2 or 3 color discrimination trials correctly (Fig. 1b). Reward was delivered 


\section{Intended}
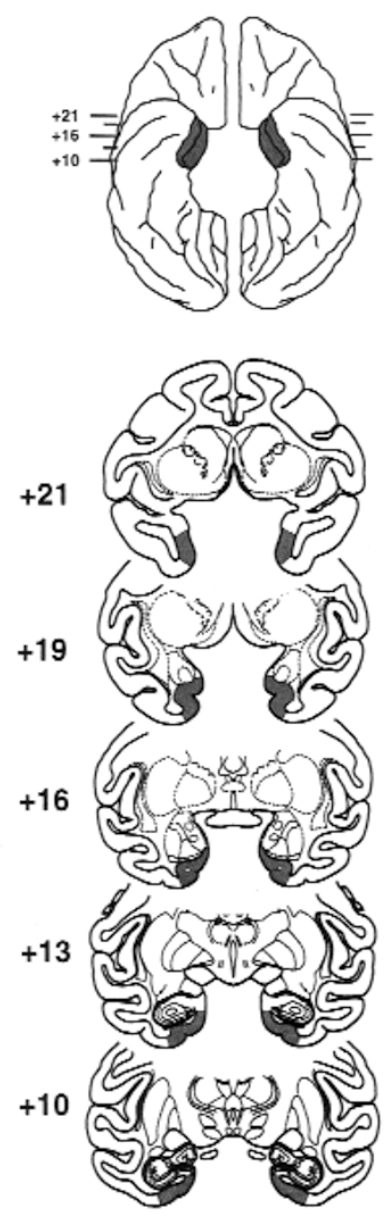

$\mathrm{Rh} 3$
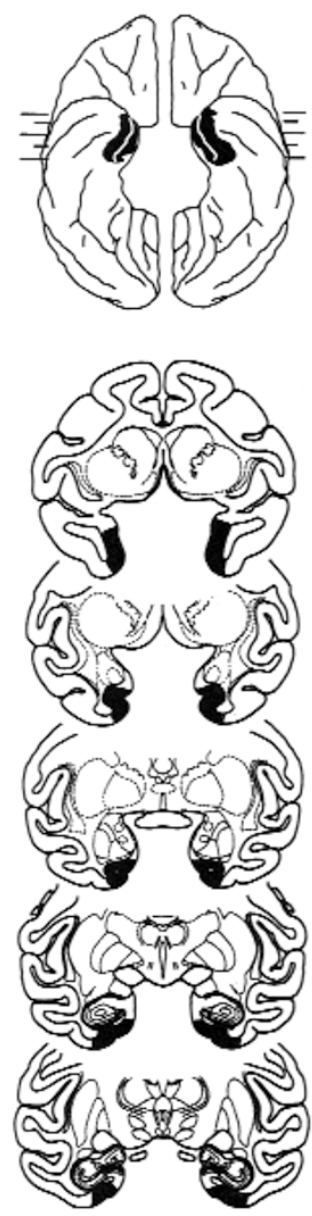

Fig 6. Extent of the rhinal cortex lesion in case Rh3. Intended lesion (gray shading), left. Plots of the lesion from MRI in case Rh3 are shown in sections at matching levels in the right column; black area, extent of the lesion. The ventral views (top) show the extent of the lesion reconstructed from the individual sections (black) and are reversed to aid in matching to the individual sections (the left hemisphere is on the left.) The numerals indicate the distance in millimeters from the interaural plane.

only at the end of a successfully completed schedule. A visual cue signaled which reward schedule was in force and which trial was current. Two sets of visual cues, brightness cues and length cues were used (Fig. 1c and $\mathbf{d}$ ). The luminance of the rectangles for the brightness cues or the lengths of the rectangles for the length cues were set in proportion to the fractional values of schedule states (the current trial number/current schedule length). As a result, each nonrewarded schedule state $(1 / 3,1 / 2$ or $2 / 3$ ) had its own distinctive cue, and each state that immediately preceded reward delivery $(1 / 1,2 / 2$ or $3 / 3)$ had the same cue.

A detailed description of the sequence of events in the task is in the Fig. 1 legend. Errors occurred when the monkeys released the lever too early or too late. There was no explicit punishment for errors; the same cue reappeared in the next trial, and the monkey still needed to complete the requisite number of correct trials for that schedule before a reward was delivered. A new schedule was picked pseudorandomly after the completion of the previous schedule. There was no requirement for the monkey to either notice or use the cue in the task.

A version of the same task, in which the cues were randomly assigned

with respect to the schedule, was also used here (shuffled condition). In the shuffled version of the task, the cue that was presented was randomly selected; therefore, the cue was no longer related to the schedule state, and no longer provided information about progress through the schedule.

Training and testing. Monkeys were initially trained to perform a color discrimination task with each correct trial being rewarded. When the monkey performed color discrimination trials at greater than or equal to $90 \%$ correct responses in two consecutive days of testing, the visually cued reward schedules (1-, 2- and 3-trial schedules) with the brightness cues were abruptly introduced.

When the monkeys' performance on this cued condition of the task was stable (2-3 days), the shuffled condition was introduced. The monkeys recognized that the cue was shuffled on the day the shuffling was introduced, and performance became stable after 1-2 days. Once performace was stable, we started switching between blocks of trials in the cued and shuffled conditions on the same testing day. Conditions would be switched without warning, and the condition used first in the daily testing session alternated between cued and shuffled conditions. Monkeys were allowed to do as many trials as they wanted, normally at the rate of 350 to 900 trials per day, for 4 days per week.

Statistics. Chi-squared test was done for each monkey using trial numbers. The linear regressions and ANOVAs were done using percent error from five or three monkeys in relation to the fractional values of the schedule states.

Surgery. After the monkeys were tested with the brightness cues, bilateral aspiration ablations of rhinal cortex were done on three monkeys. We did surgery in a single stage, as previously described ${ }^{38}$. After a twoweek recovery period, the operated monkeys resumed testing with the visually cued reward schedules.

All the experimental procedures were carried out in accordance with the National Institutes of Health Guide for the Care and Use of Laboratory Animals and were approved by the Animal Care and Use Committee of the National Institute of Mental Health.

Lesion localization. Location and extent of the cortical removal were evaluated using standard histological material for one monkey and magnetic resonance images (MRI) for all three monkeys ${ }^{22,39}$. The lesions were plotted from either digitized images of MRI scans or Nissl-stained coronal sections to drawings of a standard rhesus monkey brain at $1-\mathrm{mm}$ intervals. The volumes of the lesions, relative to the standard, were then measured using the SCION IMAGE program.

The lesions of the rhinal cortex were generally as intended. The extent of the lesion in Rh1, the one for which standard histological material is available, was measured from both the MRI and standard histology. For Rh1, damage to the rhinal cortex was estimated to be $86 \%$ and $87 \%$ from MRI and microscopic examination, respectively. Within this region, damage to the perirhinal and entorhinal cortices were estimated to be $96 \%$ and $76 \%$ from MRI, respectively, and $97 \%$ and $77 \%$ from histological material. Therefore, our estimations of the extent of the lesion from the MRI scans and Nissl-stained material are very similar.

The extent of the lesions in $\mathrm{Rh} 2$ and $\mathrm{Rh} 3$ were assessed using only MRI. Damage to the rhinal cortex was estimated to be $74 \%$ and $76 \%$ for $\mathrm{Rh} 2$ and $\mathrm{Rh} 3$, respectively. Within this region, damage to the perirhinal cortex was estimated to be $86 \%$ and $93 \%$, and damage to the entorhinal cortex was $61 \%$ and $60 \%$ for Rh 2 and Rh3, respectively. The extent of the lesion in Rh3 is shown in Fig. 6.

In each case, the damage to rhinal cortex was symmetrically distributed between left and right hemispheres. Damage to structures outside the rhinal cortex (for example, temporal cortical areas TE, TF, TH) was minor, unilateral and inconsistent across monkeys, with the exception of Rh1, in which the lesion encroached on area TE bilaterally.

\section{ACKNOWLEDgements}

This work was supported by the Intramural Research Program of the National Institute of Mental Health. 


\section{RECEIVED 13 JULY; ACCEPTED 16 OCTOBER 2000}

1. Alexander, G. E., DeLong, M. R. \& Strick, P. L. Parallel organization of functionally segregated circuits linking basal ganglia and cortex. Annu. Rev. Neurosci. 9, 357-381 (1986)

2. Mogenson, G. J., Jones, D. L. \& Yim, C. Y. From motivation to action: functional interface between the limbic system and the motor system. Prog. Neurobiol. 14, 69-97 (1980).

3. Robbins, T. W. \& Everitt, B. J. Neurobehavioral mechanisms of reward and motivation. Curr. Opin. Neurobiol. 6, 228-236 (1996).

4. Everitt, B. J., Morris, K. A., O'Brien, A. \& Robbins, T. W. The basolatera amygdala-ventral striatal system and conditioned place preference: further evidence of limbic-striatal interactions underlying reward-related processes. Neuroscience 42, 411-422 (2000).

5. Bowman, E. M., Aigner, T. G. \& Richmond, B. J. Neural signals in the monkey ventral striatum related to motivation for juice and cocaine rewards. J. Neurophysiol. 75, 1061-1073 (1996).

6. Shidara, M., Aigner, T. G. \& Richmond, B. J. Neuronal signals in the ventral striatum related to progress through a predictable series of trials. J. Neurosci. 18, 2613-2625 (1998)

7. Liu, Z. \& Richmond, B. J. Response differences in monkey TE and perirhinal cortex: stimulus association related to reward schedules. J. Neurophysiol. 83, 1677-1692 (2000)

8. Saleem, K. S. \& Tanaka, K. Divergent projections from the anterior inferotemporal area TE to the perirhinal and entorhinal cortices in the macaque monkey. J. Neurosci. 16, 4757-4775 (1996).

9. Suzuki, W. A. \& Amaral, D. G. Perirhinal and parahippocampal cortices of macaque monkey: cortical afferents. J. Comp. Neurol. 350, 497-533 (1994).

10. Mishkin, M. A memory system in the monkey. Phil. Trans. R. Soc. Lond. B Biol. Sci. 298, 83-95 (1982).

11. Baizer, J. S., Desimone, R. \& Ungerleider, L. G. Comparison of subcortical connections of inferior temporal and posterior parietal cortex in monkeys. Vis. Neurosci. 10, 59-72 (1993).

12. Saint-Cyr, J. A., Ungerleider, L. G. \& Desimone, R. Organization of visua cortical inputs to the striatum and subsequent outputs to the pallido-nigral complex in the monkey. J. Comp. Neurol. 298, 129-156 (1990).

13. Van Hoesen, G. W., Yeterian, E. H. \& Lavizzo-Mourey, R. Widespread corticostriate projections form temporal cortex of the rhesus monkey. J. Comp. Neurol. 199, 205-219 (1981).

14. Witter, M. P. \& Groenewegen, H. J. Connections of the parahippocampal cortex in the cat. IV. Subcortical afferents. J. Comp. Neurol. 252, 51-77 (1986).

15. Aggleton, D. G., Burton, M. J. \& Passingham, R. E. Cortical and subcortica afferents to the amygdala of the rhesus monkey (Macaca mulatta). Brain Res. $190,347-368(1980)$

16. Stefanacci, L., Suzuki, W. A. \& Amaral, D. G. Organization of connections between amygdaloid complex and the perirhinal and parahippocampal cortices in macaque monkeys. J. Comp. Neurol. 375, 552-582 (1996).

17. Van Hoesen, G. W. in The Amygdaloid Complex: The Differential Distribution, Diversity and Sprouting of Cortical Projections to the Amygdala in the Rhesus Monkey (ed. Ben-Ari, Y.) 77-90 (Amsterdam, Elsevier, 1981).

18. Buckley, M. J. \& Gaffan, D. Impairment of visual object-discrimination learning after perirhinal cortex ablation. Behav. Neurosci. 111, 467-475 (1997).

19. Eacott, M. J., Gaffan, D. \& Murray, E. A. Preserved recognition memory for small sets, and impaired stimulus identification for large sets, following rhinal cortex ablations in monkeys. Eur. J. Neurosci. 6, 1466-1478 (1994).
20. Gaffan, D. \& Murray, E. A. Monkeys (Macaca fascicularis) with rhinal cortex ablations succeed in object discrimination learning despite 24-hr intertrial intervals and fail at matching to sample despite double sample presentations. Behav. Neurosci. 106, 30-38 (1992).

21. Thornton, J. A., Malkova, L. \& Murray, E. A. Rhinal cortex ablations fail to disrupt reinforcer devaluation effects in rhesus monkeys (Macaca mulatta). Behav. Neurosci. 112, 1020-1025 (1998).

22. Thornton, J. A., Rothblat, L. A. \& Murray, E. A. Rhinal cortex removal produces amnesia for preoperatively learned discrimination problems but fails to disrupt postoperative acquisition and retention in rhesus moneys. J. Neurosci. 17, 8536-8549 (1997).

23. Murray, E. A., Baxter, M. B. \& Gaffan, D. Monkeys with rhinal cortex damage or neurotoxic hippocampal lesions are impaired on spatial scene learning and object reversals. Behav. Neurosci. 112, 1291-1303 (1998).

24. Suzuki, W. A. Neuroanatomy of monkey entorhinal, perirhinal and parahippocampal cortices: organization of cortical inputs and interconnections with amygdala and striatum. Semin. Neurosci. 8, 3-12 (1996).

25. Erickson, C. A. \& Desimone, R. Responses of macaque perirhinal neurons during and after visual stimulus association learning. J. Neurosci. 19, 10404-10416 (2000)

26. Higuchi, S. \& Miyashita, Y. Formation of mnemonic neuronal responses to visual paired associates in inferotemporal cortex is impaired by perirhinal and entorhinal lesions. Proc. Natl. Acad. Sci. USA 93, 739-743 (1996).

27. Miyashita. Y. Neuronal correlate of visual associative long-term memory in the primate temporal cortex. Nature 335, 817-820 (1988).

28. Miyashita, Y., Okuno, H., Tokuyama, W., Ihara, T. \& Nakajima, K. Feedback signal from medial temporal lobe mediates visual associative mnemonic codes of inferotemporal neurons. Cognit. Brain. Res. 5, 81-86 (1996).

29. Murray, E. A. in The New Cognitive Neurosciences: Memory for Objects in Nonhuman Primates (ed. Gazzaniga, M. S.) 753-763 (MIT Press, Cambridge, Massachusetts, 2000).

30. Murray, E. A., Baxter, M. G. \& Gaffan, D. Monkeys with rhinal cortex damage or neurotoxic hippocampal lesions are impaired on spatial scene learning and object reversals. Behav. Neurosci. 112, 1191-1303 (1998).

31. Murray, E. A. \& Bussey, T. J. Perceptual-mnemonic functions of the perirhinal cortex. Trends Cogn. Sci. 3, 142-151 (1999).

32. Murray, E. A. \& Gaffan, D. Removal of the amygdala plus subjacent cortex disrupts the retention of both intramodal and crossmodal associative memories in monkeys. Behav. Neurosci. 108, 494-500 (1994).

33. Murray, E. A., Gaffan, D. \& Mishkin, M. Neural substrates of visual stimulus-stimulus association in rhesus monkeys. J. Neurosci. 13, 4549-4561 (1993).

34. Parker, A. \& Gaffan, D. Lesions of primate rhinal cortex cause deficits in flavour-visual associative memory. Behav. Brain Res. 93, 99-105 (1998).

35. Sakai, K. \& Miyashita Y. Neural organization for long-term memory of paired associates. Nature 354, 152-155 (1991).

36. Sakai, K., Naya, Y. \& Miyashita, Y. Neuronal tuning and associative mechanisms in form representation. Learn. Mem. 1, 83-105 (1994).

37. Goulet, S. \& Murray, E. A. Neural substrates of crossmodal association memory in monkeys: the amygdala versus the anterior rhinal cortex. Behav. Neurosci. (in press).

38. Meunier, M., Bachevalier, J., Mishkin, M. \& Murray, E. A. Effects of visual recognition and combined and separate ablations of the entorhinal and perirhinal cortex in rhesus monkeys. J. Neurosci. 13, 5418-4332 (1993).

39. Bachevalier, J., Beauregard, M. \& Alvarado, M. C. Long-term effects of neonatal damage to the hippocampal formation and amygdaloid complex on object discrimination and object recognition in rhesus monkeys (Macaca mulatta). Behav. Neurosci. 113, 1127-1151 (1999). 